\title{
Die Rolle der Ärzteschaft im NCD-Arbeitspaket 2
}

\author{
Autoren: Dr. med. Carlos Beat Quinto ${ }^{a}, B^{2}$ arbara Weil ${ }^{b} /$ Co-Autoren: Andreas Biedermann MDc, \\ Prof. Dr. med. Jacques Cornuz ${ }^{d}$, Dr. med. Stefan Neuner-Jehle MPHe, ${ }^{\text {, }}$ unter Mitarbeit von: Dr. med. Julia \\ Dratvaf, Linda Hadorng, Dr. Fabienne Jägerf, PD Dr. med. Oskar Jennif, Dr. med. Chung-Yol Leef, \\ Dr. med. Annalis Marty-Nussbaumer ${ }^{f}$, Prof. Dr. med. Gabriela Stoppe ${ }^{f}$, Dr. med. Peter Wiedersheim ${ }^{f}$ \\ ${ }^{a}$ Mitglied des FMH-Zentralvorstandes, Departementsverantwortlicher Public Health und Gesundheitsberufe; ${ }^{b}$ Leiterin Abteilung Public Health, FMH; \\ ${ }^{c}$ PHS Public Health Services GmbH, Bern; ${ }^{d}$ Policlinique Médicale Universitaire, Université de Lausanne; ${ }^{e}$ Kollegium für Hausarztmedizin, KHM; \\ ${ }^{\dagger}$ Mitglieder Präventionskommission FMH; ${ }^{g}$ wissenschaftliche Mitarbeiterin Abteilung Public Health, FMH
}

1 In diesem Text wird der Einfachheit halber nur die männliche Form verwendet. Die weibliche Form ist selbstverständlich immer mit eingeschlossen.
Die Prävention nicht übertragbarer Krankheiten erhält in der Grundversorgung [1] in den nächsten Jahren ein neues Gewicht. Der Dialog Nationale Gesundheitspolitik, die ständige Plattform von Bund und Kantonen, und der Bundesrat verabschiedeten zu Beginn des Jahres 2016 die Strategie nichtübertragbarer Krankheiten ("non-communicable disease», NCD) [2]. Sowohl die WHO als auch die NCD-Strategie betonen die Wichtigkeit der Prävention durch die Ärzteschaft. Durch eine Verbreitung der Thematik bei uns Ärzten ${ }^{1}$ wird die Wirkung auf Bevölkerungsebene erreicht, denn wir haben einen flächendeckenden alters- und schichtenübergreifenden Zugang zur Bevölkerung und deren Vertrauen, was für die Motivation und Adhärenz von zentraler Bedeutung ist.

\section{Kernelemente des NCD-Massnahmenplans}

Die Umsetzung der NCD-Strategie erfolgt ab Januar 2017 anhand des am 24. November 2016 verabschiedeten Massnahmenplans. Zusätzlich findet am 31. Januar 2017 eine grosse Stakeholder-Konferenz statt, an der die Teilnehmenden, darunter auch Vertreter/-innen der Ärzteschaft, darüber diskutieren, wie sie zu einer effizienten Umsetzung der Strategie und ihrer Massnahmen beitragen können. Im NCD-Massnahmenplan sind folgende Stossrichtungen vorgesehen:

1. Bevölkerungsbezogene Gesundheitsförderung und Prävention - Weiterentwicklung kantonaler Programme für Gesundheitsförderung und Prävention

2. Prävention in der Gesundheitsversorgung - Optimierung der Schnittstellen zwischen Prävention und Kuration

3. Prävention in Wirtschaft und Arbeitswelt - Stärkung gesundheitsförderlicher Rahmenbedingungen in Zusammenarbeit mit der Wirtschaft

\section{NCD-Prävention über die Ärzteschaft}

Für die Ärzteschaft als relevanten Teil der Gesundheitsversorgung ist insbesondere die Massnahme 2
«Prävention in der Gesundheitsversorgung» von Bedeutung. Denn die Chancen, Patienten auf präventives Verhalten anzusprechen und zu Verhaltensänderungen zu motivieren, sind durch den Arzt am besten gegeben, suchen doch rund $80 \%$ der erwachsenen Bevölkerung mindestens einmal im Jahr einen niedergelassenen Arzt auf. Besonders hoch sind ärztliche Kontakte bei Kindern. In der Tat sind Vorsorgeuntersuchungen im Kindes- und Jugendalter zentrale Gefässe, um wichtige gesundheitsfördernde Informationen zu vermitteln und das langfristige Gesundheitsverhalten möglichst günstig zu beeinflussen. Diese Erkenntnis ist nicht neu und der Ackerboden für eine Förderung der Prävention von ärztlicher Seite ist bereits seit längerem vorbereitet [3]. Mit dem Gesundheitscoaching des KHM [4], dem Projekt Frei von Tabak [5], Paprica [6] und EviPrev [7] beispielsweise finden sich aus Sicht von Public Health bereits ausgezeichnete praxistaugliche Projekte auf einem «State of the art»-Niveau. Der Begriff «Gesundheitscoaching» hat in den letzten Jahren bereits eine interprofessionelle Ausweitung erfahren. Während beim Gesundheitscoaching die Kommunikation im Vordergrund steht und somit die Grundlage für eine effiziente Prävention in der Versorgung gelegt wird, stehen bei EviPrev die Guidelines und Dokumentationshilfen im Vordergrund. Von ärztlicher Seite her wäre es deshalb nicht nachvollziehbar, wenn diese Projekte nicht innerhalb der Massnahmen gebührend berücksichtigt würden.

\section{Schnittstelle Prävention/Kuration}

Speziell in der primären Grundversorgung ist aufgrund des biopsychosozialen Krankheitsmodells [8] Prävention ein inhärentes Thema. Natürlich sind auch Spezialisten im Bereich Prävention tätig, dies primär bei den von ihnen betreuten Krankheits- und Patientengruppen oder auf Bevölkerungsebene. Eine besondere Rolle spielen dabei die psychiatrisch, pädiatrisch 
Zusammenfassende Thesen zur Berücksichtigung bei Planung und Umsetzung der NCD-Massnahmen - nicht abschliessend

1. Ärzte sind fähig und interessiert, Gesundheitsförderungs- und Präventions-Tätigkeiten in einem interprofessionellen und integrativen Netzwerk-Ansatz auszuführen. Ärzte-Organisationen unterstützen diese Interprofessionalität, wie beispielsweise anlässlich des 2016 KHM-Jahreskongresses aufgezeigt.

2. Ärzte haben in hohem Masse Zugang zur Bevölkerung (7580\% der Schweizer Bevölkerung geht mindestens einmal im Jahr zum Arzt, Kinder sogar noch häufiger) und verfügen daher über gute Möglichkeiten, aktiv zu werden (windows of opportunity). Grundversorger beispielsweise begleiten ihre Patienten über einen langen Zeitraum und können so aktiv in Gesundheitsförderungs- und Präventions-Aktivitäten involviert sein. Auch die pädiatrischen Vorsorgeuntersuchungen sind zentral für die kontinuierliche Primär-, aber auch Sekundärprävention, um bereits in frühstem Alter die Gesundheit und das Gesundheitsverhalten positiv zu beeinflussen. Weiter können Gynäkologen anlässlich der Jahreskontrollen ebenfalls präventive Aspekte wahrnehmen. Diese Auflistung ist beliebig erweiterbar.

3. Ärzte werden von der Bevölkerung als Experten und vertrauenswürdige Akteure im Bereich Gesundheitsförderung und Prävention wahrgenommen. Aus internationaler Perspektive sind Schweizer Ärzte in der Tat in führender Position bezüglich Vertrauenswürdigkeit, wie kürzlich eine Studie von Blendon zeigte [12].

4. Ärzte verfügen über Kompetenzen und Fertigkeiten im Bereich Kommunikation, welche auf verschiedenen Ebenen der professionellen Aus-, Weiter- und Fortbildung gelehrt werden; Übermittlung und Übersetzung von Informationen

und gynäkologisch tätigen Kolleginnen und Kollegen, die oft alleinige ärztliche Betreuer ihrer Patienten sind. Impfungen wurden bisher nicht explizit erwähnt, da sie primär im Bereich übertragbarer Krankheiten eingesetzt werden. Indirekt sind sie auch im NCD-Bereich von Bedeutung, da beispielsweise gewissen Krebserkrankungen durch eine Impfung vorgebeugt werden kann. Angepasst an die Patientenbedürfnisse und im Sinne einer umfassenden Versorgung können in einer (haus-)ärztlichen Konsultation gesundheitsförderliche, primär-, sekundär-, tertiär- und quartärpräventive Aspekte [9] angesprochen werden - dies gleichzeitig mit diagnostischen, therapeutischen, rehabilitativen oder palliativen Themen. Empfehlungen müssen jedoch nicht nur vermittelt und verstanden werden, sondern auch umsetzbar sein und von einer Gesellschaft durch förderliche Verhältnisse getragen werden. An diesem Beispiel zeigt sich die im NCDArbeitspaket 2 angesprochene Schnittstelle zwischen Prävention und Kuration. Dabei muss weiter bedacht werden, dass eine wirksame Verhaltensänderung schlussendlich im täglichen Leben der Patienten erfolgt. Die ärztliche Meinung kann aber definitiv Anstoss zu dieser Veränderung geben.
(z.B. zu Risiken und Chancen) ist unabdingbar für Counselling im Bereich Gesundheitsförderung und Prävention. ÄrzteOrganisationen bieten dazu günstige Strukturen für Lehre und Ausbildung in diesen Fertigkeiten.

5. Ärzte-Organisationen bieten die bestabgestützte Evidenz, die für Abläufe im Bereich Gesundheitsförderung und Prävention vorhanden ist. Dies beispielsweise mit der Erarbeitung von Guidelines und evidenzbasierten Programmen zu Gesundheitsförderungs- und Präventionsthemen, die von der gesamten Ärzteschaft genutzt werden können.

6. Gewisse Bevölkerungsgruppen sind speziell gefährdet für NCDs: So finden sich in bildungsferneren Bevölkerungsgruppen oder manchen Migrationsgruppen z.B. vermehrt Übergewicht, schlechte Ernährung und ein höhererTabakkonsum, und Vorsorgeuntersuchungen werden eher selten in Anspruch genommen [13]. Angesichts spezifischer Bedürfnisse gewisser Bevölkerungsgruppen dürfen Adaptationen nicht vergessen werden, die es den Ärzten ermöglichen, auch diese Gruppen präventiv so gut wie möglich zu erreichen und besagten Gruppen den Zugang zu präventivem Wissen zu erleichtern (z.B. Sprachbarrieren-Abbau durch interkulturelle Dolmetscher/-innen).

7. Über die Art der finanziellen Abgeltung der ärztlichen Leistungen im Bereich Gesundheitsförderung und Prävention sollte nach Diskussion im Rahmen der Umsetzung der Massnahmen ein Konsens erreicht werden, der zwingend Berücksichtigung finden sollte.

\section{Interdisziplinäre Zusammenarbeit und partizipativer Ansatz}

Selbstredend kann die Ärzteschaft operativ nicht alle Massnahmen im Alleingang umsetzen. Der umfassende Anspruch und die systemische Sichtweise erfordern aus unserer Sicht vermehrt eine interdisziplinäre Arbeitsweise sowie die Anwendung von Methoden aus unterschiedlichen Disziplinen. Andere im Gesundheitswesen tätige Berufsgruppen und Gesundheitsligen sind in diese Netzwerke zwingend zu involvieren. In Bezug auf den optimalen Einsatz von Ressourcen ist ein qualitativ gutes Schnittstellenmanagement von ärztlicher Seite angezeigt, wofür auch Zeit und Mittel vorzusehen sind. Zudem vertreten wir den Ansatz, dass die Patientinnen und Patienten in das Zentrum der Behandlung zu stellen sind. Als gleichberechtigte Partner entscheiden diese über die Behandlung mit. Der Arzt ist Experte für das medizinische Fachwissen, dem Patienten sind seine eigenen persönlichen Werte, Wünsche und Vorstellungen bekannt. Es obliegt dabei dem Arzt, durch Herstellen einer vertrauensvollen Atmosphäre und falls notwendig durch konkretes Nachfragen den Austausch über diese Themen über- 


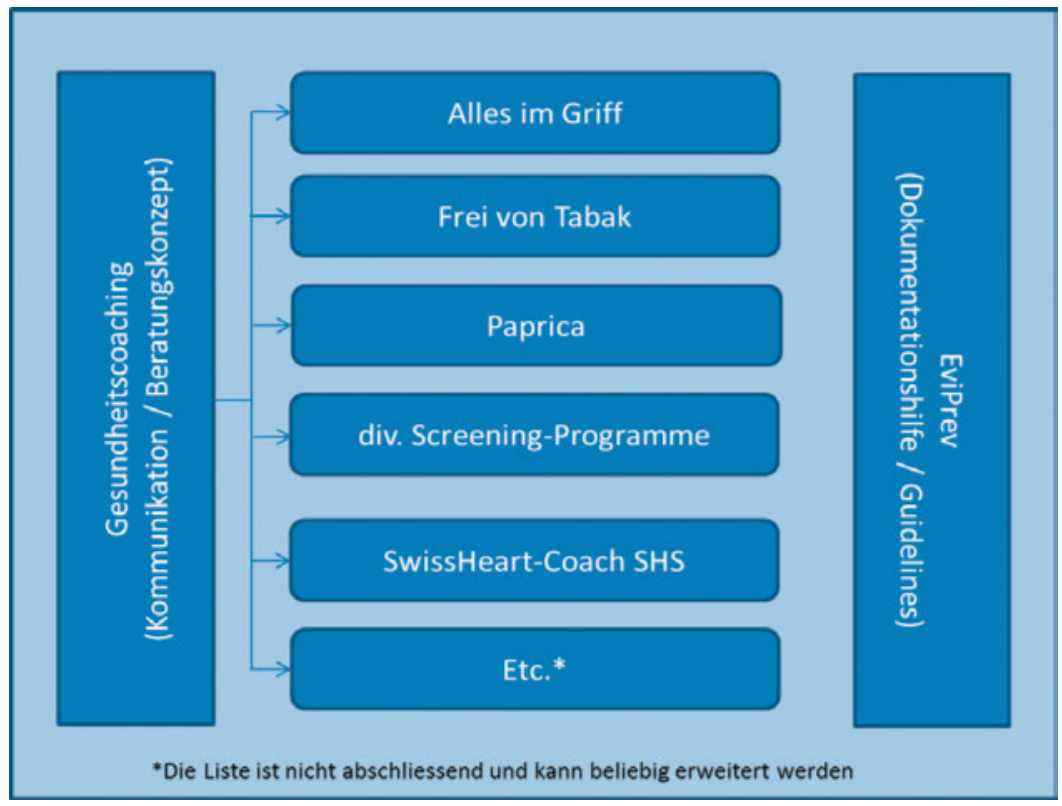

Einbettung bestehender Instrumente, Projekte und Themen - nicht abschliessend.

haupt erst zu ermöglichen. Durch diesen partizipativen Ansatz (eine Forderung seit Ottawa 1986) wäre dann auch eine entsprechende Akzeptanz bei der Umsetzung gegeben [10].

\section{Herausforderungen für die Umsetzung}

Die geplanten Massnahmen und die damit angestrebte Verbesserung werden nicht gratis zu haben sein und können nicht losgelöst von anderen gesundheitspolitischen Themen und gesellschaftlichen Voraussetzungen betrachtet werden. Dem Massnahmenplan liegt ein föderalistisches System zugrunde, das sich durch eine Vielfalt von Akteuren und Massnahmen auszeichnet, und genauso fragmentiert ist auch die Finanzierung dieses Systems. Die Ärzteschaft sieht in Bezug auf die Umsetzung der Massnahmen insbesondere folgende Herausforderungen als zentral an:

- Finanzielle Probleme, wie beispielsweise die Frage nach der Zeitentschädigung oder die fehlende Entschädigung empfohlener Untersuchungen

- Herausforderungen in Bezug auf die Motivation, Befähigung und Bildung der involvierten Akteure (Stichwort Gesundheitskompetenz)

- Interprofessionalität in der Prävention und Gesundheitsförderung als Prüfstein sowie die Gewährleistung von Schnittstellenmanagement während der gesamten Versorgungskette

Abteilung Public Health FMH - Verbindung der Schweizer Ärztinnen und Ärzte

Elfenstrasse 18

Postfach 300

CH-3000 Bern 15
Nebst dem Engagement aller Involvierten, um diesen Herausforderungen zu begegnen, braucht es Zeit für Kommunikation und Vertrauensaufbau und wenn möglich konstante Teams. Die Zukunft liegt in integrativen Ansätzen, unter Einbezug aller relevanten Akteure und unter Einhaltung der Qualität und Ethik der Standesorganisationen (Pflege, Apotheker, Ärzte). Leider wird durch das Auf-den-Markt-Drängen gewisser kommerzieller Investoren, die sich keiner Qualität und Ethik der Standesorganisationen verpflichtet fühlen, die Situation erschwert. Durch kommerzielle Investoren wird das Gesundheitspersonal in einen ethischen Konflikt gebracht: Die Investoren erwarten, dass primär das geleistet wird, was sich hinsichtlich Rendite rechnet, und nicht das, was medizinisch notwendig und sinnvoll ist. Im Rahmen der NCD-Strategie geht es darum, Bewährtes aufzunehmen, auszubauen und parallel dazu ganzheitliche Programme zu entwickeln, die sich an den Bedürfnissen der Gesellschaft orientieren, die Gesundheitsdeterminanten einbeziehen und finanziell gesichert sind. Trotz allen Herausforderungen, die uns in Hinsicht auf die Umsetzung noch erwarten, sind wir bestrebt, unseren Einsatz zu leisten. Wir hoffen auf eine gute Zusammenarbeit und ein Voranbringen der NCD-Strategie mit allen anderen in der Gesundheitsversorgung tätigen Professionen [11].

\section{Bildnachweis}

(c) Abteilung Public Health

\section{Literatur}

1 Grundversorgung wird in diesem Artikel umfassend verstanden (Präventionsleistungen aus der primären, sekundären, tertiären und quartären Versorgung).

2 www.bag.admin.ch/ncd

3 MedBG, 2. Abschnitt, Art 8, h: Ärzte «setzen sich für die menschliche Gesundheit ein, indem sie beratend tätig sind und die erforderlichen präventiven und gesundheitsfördernden Massnahmen in ihrem Berufsfeld treffen» / Lernzielkatalog SIWF: «Der Facharzt nutzt die Gelegenheiten für Beratungen bezüglich der Erhaltung der Gesundheit und der Gesundheitsförderung.»

4 http://www.gesundheitscoaching-khm.ch/

5 http://www.frei-von-tabak.ch/

6 http://www.paprica.ch/

7 http://www.public-health-services.ch/index-de.php?frameset=120

8 "Das erweiterte biopsychosoziale Modell ist eine fundamentale Theorie der Körper-Seele-Einheit und ermöglicht erstmals ein wissenschaftlich begründetes ganzheitliches Verständnis von Krankheit bzw. Gesundheit.» Egger, JW. Grundlagen der Psychosomatik Zur Anwendung des biopsychosozialen Krankheitsmodells in der Praxis. Psychologische Medizin. 2008;19(2):12-22.

9 «Quartäre Prävention bedeutet die Verhinderung von Übermedikalisierung und unnützer Medizin». M. Jamoulle. Quaternary prevention, an answer of family doctors to overmedicalization. International Journal of Health Policy and Management. 2015;4,4:1-4.

10 Auswahl an Instrumenten: Motivational Interviewing, Shared Decision Making, Informed Consent.

11 Anmerkung zu der Formulierung: Die Bezeichnungen Medizinalund Gesundheitsberufe sind durch den Gesetzgeber belegt und umfassen längst nicht alle Berufe, die im Gesundheitswesen tätig sind.

12 N. Engl, J Med 2015.

13 Zweites Gesundheitsmonitoring der Migrationsbevölkerung in der Schweiz (GMM II). 